\title{
A Negative Mass Theorem for Surfaces of Positive Genus
}

\author{
K. Okikiolu ${ }^{\star}$ \\ Department of Mathematics, University of California, San Diego, \\ 9500 Gilman Drive, La Jolla, CA 92093, USA. E-mail: okikiolu@math.ucsd.edu
}

Received: 5 October 2008 / Accepted: 13 October 2008

Published online: 22 January 2009 - (C) The Author(s) 2009. This article is published with open access at Springerlink.com

\begin{abstract}
Let $M$ be a closed surface. For a metric $g$ on $M$, denote the Laplace-Beltrami operator by $\Delta=\Delta_{g}$. We define trace $\Delta^{-1}=\int_{M} m(p) d A$, where $d A$ is the area element for $g$ and $m(p)$ is the Robin constant at the point $p \in M$, that is the value of the Green function $G(p, q)$ at $q=p$ after the logarithmic singularity has been subtracted off. Since trace $\Delta^{-1}$ can also be obtained by regularization of the spectral zeta function, it is a spectral invariant. Heuristically it represents the sum of squares of the wavelengths of the surface. We define the $\Delta$-mass of $(M, g)$ to equal (trace $\left.\Delta_{g}^{-1}-\operatorname{trace} \Delta_{S^{2}, A}^{-1}\right) / A$, where $\Delta_{S^{2}, A}$ is the Laplacian on the round sphere of area $A$. This is an analog for closed surfaces of the ADM mass from general relativity. We show that if $M$ has positive genus, the minimum of the $\Delta$-mass on each conformal class is negative and attained by a smooth metric. For this minimizing metric, there is a sharp logarithmic Hardy-LittlewoodSobolev inequality and a Moser-Trudinger-Onofri type inequality.
\end{abstract}

\section{Section 1. Introduction}

Let $M$ be a closed Riemann surface and let $g$ be a metric on $M$ compatible with the complex structure. With respect to complex coordinates $z$, the metric $g$ is the real part of the Kähler metric

$$
e^{u} d z \otimes d \bar{z}
$$

for some smooth real valued function $u$ on $M$. The area element is

$$
d A=\frac{i e^{u}}{2} d z \wedge d \bar{z} .
$$

Denote the total area by $A$. The Laplace Beltrami operator for the metric $g$ is given by

$$
\Delta=\Delta_{g}=-4 e^{-u} \partial_{z} \partial_{\bar{z}}
$$

\footnotetext{
* The author would like to acknowledge the support of the Institute for Advanced Study.
} 
(This is sometimes called the geometer's Laplacian: note the sign.) The Green's function for the metric $g$ is the smooth real valued function $G$ on $M \times M \backslash\{(p, p): p \in M\}$ such that

$$
\int_{M} G(p, q) \Delta f(q) d A(q)=f(p)-\frac{1}{A} \int_{M} f d A
$$

for smooth functions $f$ on $M$. It follows that $G$ is symmetric, $\int G(p, q) d A(q)=0$, and

$$
\Delta_{p} G(p, q)=-\frac{1}{A} \quad \text { when } p \neq q
$$

For the smooth function $f$ on $M$ we define

$$
\Delta^{-1} f(p)=\int_{M} G(p, q) f(q) d A(q) .
$$

If $d(p, q)$ is the geodesic distance between $p$ and $q$ in the metric $g$ then there exists a smooth function $m$ on $M$ such that

$$
G(p, q)=\frac{1}{2 \pi} \log d(p, q)+m(p)+O(d(p, q)), \quad \text { as } d(p, q) \rightarrow 0 .
$$

The value $m(p)$ is known as the Robin constant at $p$. We define

$$
\operatorname{trace} \Delta_{g}^{-1}=\int_{M} m_{g} d A
$$

This is a spectral invariant for $\Delta$, since it can be obtained from the spectral zeta function associated to $\Delta$, see [S1,S2, M3], or [Ok1]. Heuristically it represents the sum of squares of the wavelengths of the surface (up to a constant). It is convenient to normalize to get a scale invariant quantity. Indeed, define the $\Delta$-mass to be

$$
\mathcal{M}(g)=\frac{\operatorname{trace} \Delta_{g}^{-1}-\operatorname{trace} \Delta_{S^{2}, A}^{-1}}{A},
$$

where $\Delta_{S^{2}, A}$ is the Laplacian for the round metric on $S^{2}$ with area $A$. Then the above results show that $\mathcal{M}(g)$ is always positive when $g$ is a metric on $S^{2}$. In this paper we show the following:

Theorem 1 (Negative mass theorem for positive genus surfaces). Given a metric $g$ on the closed surface $M$ of positive genus, there exists a conformal metric $e^{\phi} g$ such that $\mathcal{M}\left(e^{\phi} g\right)<0$. In fact $e^{\phi} g$ can be chosen to minimize $\mathcal{M}$ within the conformal class.

When $M$ is a torus, this was proved in [Ok2]. We remark that Theorem 1 fails on the sphere. This follows from the logarithmic Hardy-Littlewood-Sobolev inequality for the sphere [On, CL, B]:

Theorem (Morpurgo [M2]) For a metric $g$ on the sphere $S^{2}$, the value $\mathcal{M}(g)$ is strictly positive unless $g$ is round.

From [Ok1], we immediately obtain the following corollary to Theorem 1 
Corollary 2 (Analogs of Logarithmic HLS inequality and the Moser-Trudinger-Onofri Inequality for general surfaces). If the metric $g$ minimizes $\mathcal{M}$ within its conformal class, then

$$
\frac{1}{4 \pi} \int_{M} \psi e^{\psi} d A-\frac{1}{A} \int_{M} e^{\psi} \Delta^{-1} e^{\psi} d A \geq 0
$$

for all functions $\psi: M \rightarrow \mathbb{R}$ with $\int_{M} e^{\psi} d A=A$ such that $\int_{M} \psi e^{\psi} d A$ is finite. Here, $d A$ and $\Delta$ are associated to $g$. Moreover, for $\psi \in C^{\infty}(M)$,

$$
\frac{1}{16 \pi} \int_{M} \psi \Delta \psi d A-\log \left(\frac{1}{A} \int_{M} e^{\psi} d A\right)+\frac{1}{A} \int_{M} \psi d A \geq 0 .
$$

For some related results, see [Ch,CheC,DJLW,LL1,LL2,M2,M3,NT,Ok1,Ok2,OPS, S2].

Remark. The quantity $\mathcal{M}(g)$ can be viewed as an analog of the ADM mass. Indeed, writing $K(p)$ for the Gaussian curvature of $g$ at $p$, it is shown in [S1,S2], that for any metric $g$ on the 2-sphere, the natural analog of the ADM mass for metrics on the sphere is the constant

$$
m_{g}(p)-\frac{1}{2 \pi} \Delta^{-1} K(p)=\frac{1}{A} \operatorname{trace} \Delta_{g}^{-1} .
$$

Although the left-hand side of (1.2) is not constant in general for surfaces of higher genus, the right-hand side can be thought of as the analog of the mass. (The left-hand side of (1.2) is constant for the canonical metric, a fact we use in next section.) For a probabilistic interpretation of trace $\Delta^{-1}$, see [DS1]. There it is shown that trace $\Delta^{-1}$ is the constant term in an asymptotic expansion in $\varepsilon$, of the time it takes a Brownian particle starting at a randomly chosen point on the surface to get $\varepsilon$-close to another randomly chosen point.

\section{Section 2. The Proof}

There are two main ingredients in the proof of this result. The first is an identity concerning the Arakelov Green's function which is used in the construction of the Arakelov metric. The second is a delicate result on the mean field equation on surfaces proved in [DJLW]. That paper gives conditions under which a general mean field equation has a solution. Here we show that for the particular case of the canonical metric on $M$ and the mean field equation arising from trace $\Delta^{-1}$, the conditions of the [DJLW] theorem are satisfied. We start by recalling the way that Robin's constant and the sum of squares of the wavelengths change under a conformal change of the metric.

Proposition 2.1. Conformal change of the Robin constant. If $\phi$ is a smooth function on $M$ then

$$
m_{e^{\phi} g}(p)=m_{g}(p)+\frac{\phi}{4 \pi}-\frac{2}{A_{\phi}}\left(\Delta_{g}^{-1} e^{\phi}\right)(p)+\frac{1}{A_{\phi}^{2}} \int_{M} e^{\phi} \Delta_{g}^{-1} e^{\phi} d A,
$$

where

$$
A_{\phi}=\int_{M} e^{\phi} d A
$$

For the proof, see for example [S1,S2,M3] or [Ok1]. 
Proposition 2.2. Conformal change of trace $\Delta^{-1}$ (Morpurgo's Formula) If $\phi$ is a smooth function on $M$, then

$$
\operatorname{trace} \Delta_{e^{\phi} g}^{-1}=\int_{M} m_{g} e^{\phi} d A+\frac{1}{4 \pi} \int_{M} \phi e^{\phi} d A-\frac{1}{A_{\phi}} \int_{M} e^{\phi} \Delta_{g}^{-1} e^{\phi} d A .
$$

Now we discuss the Canonical metric and the Arakelov Green's function. We refer the reader to [W] and [F] for more details on this subject. If $M$ is a Riemann surface of genus $H$, there exists a metric $g$ on $M$ known as the canonical metric which is compatible with the complex structure. It is defined by taking the Jacobian embedding of the Riemann surface $M$ into a $2 H$-dimensional torus, and pulling back the flat metric on the torus to $M$. Indeed, let $\left\{A_{j}, B_{j}\right\}$ be a symplectic homology basis for $H_{1}(M, \mathbb{Z})$ satisfying the intersection pairings

$$
\#\left[A_{i}, A_{j}\right]=0, \quad \#\left[B_{i}, B_{j}\right]=0, \quad \#\left[A_{i}, B_{j}\right]=\delta_{i j} .
$$

Take a basis $\theta_{j}$ for the space of homomorphic 1-forms satisfying

$$
\int_{A_{j}} \theta_{k}=\delta_{j k}
$$

Then the period matrix $\Omega_{i j}$ given by

$$
\Omega_{i j}=\int_{B_{i}} \theta_{j}
$$

is positive definite. The Jacobian variety associated to $M$ is

$$
J(M)=\mathbb{C}^{H} /\left(\mathbb{Z}^{h}+\Omega \mathbb{Z}^{H}\right) .
$$

The Abel map gives an embedding of $M$ into $J(M)$,

$$
I: z \rightarrow \int_{z_{0}}^{z}\left(\theta_{1}, \ldots, \theta_{H}\right)
$$

The canonical Kähler metric on $M$ is given in terms of local holomorphic coordinates $z$ by

$$
\mu(z) d z \otimes d \bar{z}, \quad \text { where } \quad \mu(z)=\frac{1}{H}\left(\sum_{j, k=1}^{H}(\operatorname{Im} \Omega)_{j k}^{-1} \frac{d \theta_{j}}{d z} \frac{d \bar{\theta}_{k}}{d \bar{z}}\right)
$$

The real part of the Kähler metric is the Riemannian metric $g$. It can be checked that this metric has unit area. The Green's function for this metric is known as the Arakelov Green's function and the following result is well known.

Proposition 2.3. If $M$ is a closed Riemann surface of genus $H$ and if $g$ is the canonical metric on $M$ with unit area, then the Robin constant $m(p)$ for $g$ satisfies

$$
\Delta m(p)=2 H-2+\frac{K(p)}{2 \pi} .
$$


Proof of Proposition 2.3. The Gaussian curvature $K$ is given by

$$
2 \partial_{z} \partial_{\bar{z}} \log \mu=-\mu K .
$$

The Arakelov Green's function is given by

$$
\begin{aligned}
G(z, w)= & -\frac{1}{2 \pi} \log |E(z, w)|+\frac{1}{2} \sum_{j, k=1}^{H}(\operatorname{Im} \Omega)_{j k}^{-1} \operatorname{Im}(Z-W)_{j} \operatorname{Im}(Z-W)_{k} \\
& +\frac{m(z)}{2}-\frac{\log \mu(z)}{8 \pi}+\frac{m(w)}{2}-\frac{\log \mu(w)}{8 \pi} .
\end{aligned}
$$

Here, $Z=I(z)$ and $W=I(w)$ and $E(z, w)$ is the prime form which plays the role of $z-w$, is holomorphic in $z$ and $w$, and transforms as a $(-1 / 2,-1 / 2)$ form in each variable. We notice that $\log |E(z, w)|$ is harmonic, and so from (1.1) we have that for $w \neq z$

$$
\mu(z)=4 \partial_{z} \partial_{\bar{z}} G(z, w)=H \mu(z)+2 \partial_{z} \partial_{\bar{z}} m(z)+\frac{\mu(z) K(z)}{4 \pi} .
$$

Hence we see that

$$
4 \mu^{-1} \partial_{z} \partial_{\bar{z}} m=2-2 H-\frac{K}{2 \pi}
$$

Theorem 1 is now an application of the following result on the mean field equation which is obtained from Theorem 1.2 of [DJLW] and its proof.

Theorem 2.4. [DJLW] Let $(M, g)$ be a closed surface of unit area and let $h$ be a smooth positive function on $M$. Suppose $p_{0}$ is a point at which $8 \pi m+2 \log h$ attains its maximum value, and suppose in addition that

$$
\Delta \log h\left(p_{0}\right)<8 \pi-2 K\left(p_{0}\right) .
$$

Then the minimum of the functional

$$
J(u)=\frac{1}{16 \pi} \int_{M}|\nabla u|^{2} d A+\int_{M} u d A-\log \int_{M} h e^{u} d A
$$

over functions $u$ in the Sobolev space $H^{1}(M)$ is attained at a smooth function u satisfying

$$
\Delta u=8 \pi h e^{u}-8 \pi .
$$

Moreover, for this minimum point $u$ we have

$$
J(u)<-\left(1+\log \pi+\max _{p \in M}\left(4 \pi m_{g}(p)+\log h(p)\right)\right) .
$$

Proof of Theorem 1. We take $g$ to be the canonical metric on $M$, and we set

$$
h=e^{-4 \pi m_{g}} .
$$


Then by Proposition 2.3, we have

$$
\Delta \log h=-4 \pi \Delta m_{g}=8 \pi-8 \pi H-2 K<8 \pi-2 K .
$$

Hence we obtain the conclusion of Theorem 2.4 From (2.3), the function $u$ satisfies

$$
\int_{M} h e^{u} d A=1
$$

From (2.2) and (2.3) we see that

$$
J(u)=\frac{1}{2} \int_{M} u\left(h e^{u}+1\right) d A .
$$

However, writing

$$
\phi=u-4 \pi m_{g}
$$

we have

$$
\int_{M} e^{\phi} d A=1
$$

and

$$
\Delta_{g}^{-1} e^{\phi}=\frac{1}{8 \pi}\left(u-\int_{M} u\right)
$$

Hence from (2.1),

$$
\operatorname{trace} \Delta_{e^{\phi} g}^{-1}=\frac{1}{8 \pi} \int_{M} u\left(h e^{u}+1\right)=\frac{J(u)}{4 \pi} .
$$

Now from (2.4) and the fact that

$$
\operatorname{trace} \Delta_{S^{2}, 1}^{-1}=\frac{-1-\log \pi}{4 \pi},
$$

we see that

$$
\operatorname{trace} \Delta_{e^{\phi} g}^{-1}<\operatorname{trace} \Delta_{S^{2}, 1}^{-1} .
$$

In fact we remark that $e^{\phi} g$ minimizes trace $\Delta^{-1}$ among unit area metrics in the conformal class of $g$. Indeed, from [Ok] Theorem 1, we conclude that the minimum of trace $\Delta_{e^{\psi} g}^{-1}$ among conformal factors with $\int e^{\psi}=1$, must in fact be attained at a metric $e^{\psi} g$ with $\int_{M} e^{\psi} d A=1$, which must also satisfy the Euler-Lagrange equation, namely that the Robin constant $m_{e^{\psi}}(p)$ is constant:

$$
\Delta_{g}^{-1} e^{\psi}=\frac{1}{8 \pi}\left(\psi+4 \pi m_{g}-\int_{M}\left(\psi+4 \pi m_{g}\right) d A\right) .
$$

However, setting $v=\psi+4 \pi m_{g}$, we find that $v$ is a critical metric for $J$, and hence

$$
\operatorname{trace} \Delta_{e^{\psi} g}^{-1}=\frac{J(v)}{4 \pi} \geq \frac{J(u)}{4 \pi}=\operatorname{trace} \Delta_{e^{\phi} g}^{-1} .
$$

We also remark that in general the metric $e^{\phi} g$ need not coincide with the canonical metric or the constant curvature metric or the Arakelov metric, see [Ok2]. On a long thin rectangular torus, the minimizer is close to being a round sphere with a short worm hole joining the poles. 
Acknowledgement. I would like to thank Richard Wentworth for helpful discussions.

Open Access This article is distributed under the terms of the Creative Commons Attribution Noncommercial License which permits any noncommercial use, distribution, and reproduction in any medium, provided the original author(s) and source are credited.

\section{References}

[B] Beckner, W.: Sharp sobolev inequalities on the sphere and the moser-trudinger inequality. Annals of Math. 138, 213-242 (1993)

[CL] Carlen, E., Loss, M.: Competing symmetries, the logarithmic hls inequality and onofri's inequality on $s^{n}$. Geom. Funct. Anal. 2, 90-104 (1992)

[Ch] Chang, S.-Y.A.: Conformal invariants and partial differential equations. Bull. Amer. Math. Soc. 42, 365-393 (2005)

[CheC] Chen, C.-C., Lin, C.-S.: Sharp estimates for solutions of multi-bubbles in compact riemann surfaces. Comm. Pure Appl. Math. 55(6), 728-771 (2002)

[DJLW] Ding, W., Jost, J., Li, J., Wang, G.: The differential equation $\Delta u=8 \pi-8 \pi h e^{u}$ on a compact riemann surface. Asian J. Math. 1, 230-248 (1997)

[DS1] Doyle, P., Steiner, J.: Spectral invariants and playing hide and seek on surfaces. Preprint, available at http://www.cims.nyu.edu/ steiner/hideandseek.pdf, 2005

[DS2] Doyle, P., Steiner, J.: Blowing bubbles on the torus. Preprint, available at http://www.cims.nyu.edu/ $\sim$ steiner/torus.pdf, 2005

[F] Fay, T.: Theta functions on riemann surfaces. Ann. Math. 119, 387 (1994)

[LL1] Lin, C.-S., Lucia, M.: Uniqueness of solutions for a mean field equation on the torus. J. Diff. Eqs. 229(1), 172-185 (2006)

[LL2] Lin, C.-S., Lucia, M.: One-dimensional symmetry of periodic minimizers for a mean field equation. Ann. Sc. Norm. Super. Pisa Cl. Sci. (5) 6(2), 269-290 (2007)

[M1] Morpurgo, C.: The logarithmic hardy-littlewood-sobolev inequality and extremals of zeta functions on $s^{n}$. Geom. Funct. Anal. 6, 146-171 (1996)

[M2] Morpurgo, C.: Zeta functions on $S^{2}$. Extremal Riemann surfaces (San Francisco, 1995), Contemp. Math., 201, Providence, RI: Amer. Math. Soc., 1997, pp. 213-225

[M3] Morpurgo, C.: Sharp inequalities for functional integrals and traces of conformally invariant operators. Duke Math. J. 114, 477-553 (2002)

[NT] Nolasco, M., Tarantello, G.: On a sharp sobolev-type inequality on two-dimensional compact manifolds. Arch. Ration. Mech. Anal. 145, 161-195 (1998)

[Ok1] Okikiolu, K.: Extremals for logarithmic hls inequalities on compact manifolds.. GAFA 107(5), 1655-1684 (2008)

[Ok2] Okikiolu, K.: A negative mass theorem for the 2-torus. Commun. Math. Phys. 284(3), 775-802 (2008)

[On] Onofri, E.: On the positivity of the effective action in a theory of random surfaces. Commun. Math. Phys. 86, 321-326 (1982)

[OPS] Osgood, B., Phillips, R., Sarnak, P.: Extremals of determinants of laplacians. J. Funct. Anal. 80, 148-211 (1988)

[S1] Steiner, J.: Green's Functions, Spectral Invariants, and a Positive Mass on Spheres. Ph. D. Dissertation, University of California San Diego, June 2003

[S2] Steiner, J.: A geometrical mass and its extremal properties for metrics on $s^{2}$. Duke Math. J. 129, 63-86 (2005)

[W] Wentworth, R.: The asymptotics of the arakelov-green's function and faltings' delta invariant. Commun. Math. Phys. 137, 427-459 (1991)

Communicated by S. Zelditch 